\title{
Análise comparativa da assembléia de aves em dois remanescentes florestais no interior do Estado de São Paulo, Brasil
}

\author{
Reginaldo J. Donatelli ${ }^{\text {; }}$ Carolina D. Ferreira ${ }^{2}$; Andreli C. Dalbeto ${ }^{2} \&$ Sérgio R. Posso ${ }^{3}$ \\ 1 Departamento de Ciências Biológicas, Faculdade de Ciências, Universidade Estadual Paulista. Caixa Postal 473, \\ 17001-970 Bauru, São Paulo, Brasil. E-mail: rjdonat@fc.unesp.br \\ 2 Departamento de Zoologia, Instituto de Biociências, Universidade Estadual Paulista. Caixa Postal 510, \\ 18618-000 Botucatu, São Paulo, Brasil. E-mail: fdcarol@yahoo.com.br \\ ${ }^{3}$ Departamento de Ciências Biológicas, Universidade Federal do Mato Grosso. 78735-901 Rondonópolis, Mato Grosso, Brasil.
}

\begin{abstract}
Comparative analysis of birds community in two forested fragments in the State of São Paulo, Brazil. Qualitative and quantitative survey of bird community were performed in two distinct semideciduous forest in the interior of the State of São Paulo from July 2004 to July 2005. Point Counts were used for the quantitative survey followed by diversity and frequence of ocurrence indexes of the bird assemblage. The qualitative survey registered a total of 181 species of birds at Fazenda Rio das Pedras (FRP, Itapetininga, 350 ha) and 126 at Fazenda Santa Maria II (FSM II, Buri, 260 ha). The qualitative survey registered 73 species in 998 contacts and 64 species en 1019 contacts, respectively, for FRP and FSM II. The ponctual index of abundance (IPA) varied from 0.01 (one contact) to 1.32 (132 contacts) at FRP and from 0.01 (one contact) to 0.97 (97 contacts) at FSM II. The general diversity index for FRP was $\mathrm{H}^{\prime}=2.85$ and $\mathrm{H}^{\prime}=3.04$ at FSM II. Both areas presented an evenness index of $E=0.91$. The bird community in both patched of forest showed the same pattern found in other different forest fragments of relative size studies by many distinct authors. In both localities, insectivorus (53\% at FSM e 50\% at FRP) and frugivorus ( $23 \%$ na FSM e $26 \%$ na FRP) birds were the most representative. Among the former, Tyrannidae and Thamnophilidae were predominant, respectively, at FSM and FRP. In both places understory insectivorous birds were dominant (53\% at FSM and $51,4 \%$ at FRP), followed by understory frugivorus at FSM (50\%) and by canopy frugivorus $(52,6 \%)$ at FRP. The importance of bird community in forest remmants in linked to managing and conservation of wild natural forested in Stade of São Paulo.
\end{abstract}

KEY-WORDS. Bird structure; forest remmants; trophic structure.

RESUMO. Realizou-se o levantamento quantitativo e qualitativo da comunidade de aves de dois fragmentos de floresta estacional semidecídua no interior do estado de São Paulo de julho de 2004 a julho de 2005. Para o estudo quantitativo utilizou-se da metodologia de Pontos de Escuta. Foram analisados os índices de diversidade e de frequiência de ocorrência dessa comunidade. O levantamento qualitativo registrou 181 espécies na Fazenda Rio das Pedras - FRP (Itapetininga, 350 ha) e 126 espécies na Fazenda Santa Maria II - FSM (Buri, 480 ha), enquanto que o levantamento quantitativo registrou a presença de 73 espécies em 988 contatos e 64 espécies em 1019 contatos para FRP e FSM, respectivamente. O índice pontual de abundância (IPA) variou de 0,01 (1 contato) a 1,32 (132 contatos), para FRP e na FSM variou entre 0,01 (1 contato) a 0,97 (97 contatos). A diversidade do fragmento da FRP foi de $\mathrm{H}^{\prime}=3,04$ e na FSM de $\mathrm{H}^{\prime}=2,85$ onde a eqüitatividade em ambas áreas foi de 0,91 . A comunidade de aves nos fragmentos estudados mostrou o mesmo padrão encontrado em outros fragmentos de floresta estacional semidecídua de tamanhos relativos. As categorias alimentares mais representativas nos dois remanescentes foram insetívoras ( $53 \%$ na FSM e $50 \%$ na FRP) e frugívoras (23\% na FSM e $26 \%$ na FRP). Dentre os insetívoros, destacaram-se as famílias Tyrannidae na FSM e Thamnophilidae na FRP. Tanto na FSM como na FRP os insetívoros de sub-bosque foram mais representativos ( $53 \%$ e $51,4 \%$ respectivamente), seguidos pelos frugívoros de sub-bosque (50\%) na FSM e frugívoros de copa $(52,6 \%)$ na FRP. A importância do estudo de comunidade de aves esta ligada à elaboração do plano de manejo e conservação das áreas naturais.

PALAVRAS-CHAVE. Avifauna; comunidade; fragmentos; categorias alimentares.

De acordo com um levantamento recém-concluído do Instituto Florestal de São Paulo, restam apenas 13,9\% da co- bertura vegetal natural paulista, o equivalente a 3,46 milhões de hectares (ZoRzetTo et al. 2003). Curiosamente, este percentual 
corresponde à área de vegetação natural derrubada durante um dos mais intensos períodos de devastação, (década de 60), quando ainda não havia preocupação com danos ambientais.

Cerca de 40\% das espécies de aves no Novo Mundo são ameaçadas, principalmente devido à perda de habitat, sendo que mais de $8 \%$ são prejudicadas exclusivamente por possuírem área de ocorrência restrita (Collar et al. 1992). A fragmentação de habitats pode ter diversos efeitos sobre a comunidade de aves, mas alguns indivíduos podem não responder imediatamente a essas alterações (TEMPLE \& WiENs 1989), o que pode retardar a detecção dos efeitos da fragmentação (Bierregaard et al. 1992). Assim, ela reduz a área viável para a sobrevivência das espécies sensíveis bem como a sua biodiversidade (JARVIS 1994, BIERREGAARD \& Lovejoy 1989) e, conseqüentemente, modifica a composição das comunidades e a densidade populacional (VERNER 1981).

A discussão de quanto a fragmentação do habitat e a modificação das comunidades biológicas podem ser toleradas pelas espécies, sem haver perda da abundância ou de funções biológicas, torna-se um ponto crucial no estudo das comunidades de aves (DANielsen 1997).

Habitats fragmentados afetam negativamente a sobrevivência das populações e a diversidade biológica, sendo essa a primeira consideração para iniciar uma estratégia de conservação (Wilcox \& Murphy 1985).

Este estudo teve por objetivos caracterizar a avifauna em dois fragmentos de floresta estacional semidecídua no Estado de São Paulo, analisar e comparar a estrutura da comunidade de aves dos fragmentos por meio de dados qualitativos como a composição específica e freqüência de ocorrência; analisar e comparar quantitativamente a comunidade de aves dos fragmentos por meio dos índices de abundância e de diversidade e da utilização de recursos alimentares e ocupação de estratos vegetais.

\section{MATERIAL E MÉTODOS}

\section{Área de estudo}

O presente estudo foi realizado em dois fragmentos de floresta estacional semidecídua nas Fazendas Rio das Pedras $\left(23^{\circ} 23^{\prime} \mathrm{S}, 48^{\circ} 36^{\prime} \mathrm{W}\right)$, Itapetininga, e Santa Maria II (23⒌ $51^{\prime} \mathrm{S}$, $48^{\circ} 42^{\prime}$ W), Buri, Estado de São Paulo.

A área da Fazenda Rio das Pedras (FRP) é de 4.668,38 ha e o fragmento estudado possui 350ha circundado por cultivo de eucalipto. Este fragmento apresenta várias clareiras no seu interior, com muitas lianas e bambus. A fazenda apresenta ambientes antrópicos como lagoas, bosques, jardins, pomares e uma grande represa bem próxima ao fragmento estudado. A maior parte da fazenda é utilizada para o plantio de eucalipto.

A Fazenda Santa Maria II (FSM) possui $3.478,88$ ha e o fragmento de estudo possui 480 ha circundado por cultivo de eucalipto. Este fragmento apresenta uma fisionomia vegetal diferente do fragmento da Fazenda Rio das Pedras, com elementos vegetais de grande porte e com poucas clareiras no seu interior. Outros ambientes também aparecem na fazenda, como bosques, jardins e pomares.

\section{Metodologia}

Os métodos para os levantamentos qualitativos e quantitativos seguem Donatell et al. (2004). No levantamento qualitativo foram realizadas 10 visitas em cada área de estudo. $\mathrm{O}$ esforço amostral foi de oito horas de observação/visita, perfazendo um total de 80 horas de observação. As espécies foram identificadas visualmente e/ou por meio auditivo, levando-se em consideração informações gerais sobre alimentação e estrato principal de forrageamento. Este estudo cobriu também as áreas adjacentes ao fragmento nas duas fazendas.

O levantamento quantitativo ocorreu entre julho de 2004 a julho de 2005, período em que foram estudados 100 pontos com duração de 10 minutos cada e ficou restrito exclusivamente aos fragmentos. Foram utilizadas trilhas pré-existentes nas matas que variavam de 800 a 1200 m na FRP e de 1000 a 2000 m na FSM.

A freqüência de ocorrência (F.O.), a abundância específica e o índice de diversidade seguiram Donatell et al. (2004). O índice de similaridade utilizado foi o de Sorensen (Krebs 1999).

O estudo das categorias tróficas ficou restrito exclusivamente ao interior dos fragmentos nas duas áreas amostrais. Para as categorias alimentares foi seguida a proposta de MotTA-JúNIOR (1990).

Considerou-se neste estudo o número de registros ou de indivíduos (contatos) para a estruturação das relações tróficas e não somente o número de espécies em cada categoria de dieta. Este tipo de análise nos fornece uma representação mais real do uso dos recursos alimentares e em conseqüência, uma nítida relação entre determinados tipos de dieta e habitat (MotTa-JúNior 1990). Para a classificação da ocupação das espécies no estrato vegetal foram considerados cinco estratos, a saber: solo, sub-bosque, copa, vertical e aquático.

\section{RESULTADOS E DISCUSSÃO}

\section{Levantamento Qualitativo}

No levantamento qualitativo foram registradas 180 espécies de aves distribuídas em 49 famílias na Fazenda Rio das Pedras (FRP, daqui em diante) e 126 espécies em 39 famílias, na Fazenda Santa Maria II (FSM, daqui em diante) (Anexo I).

As famílias mais representativas da ordem Passeriformes em números de espécies na FRP foram Tyrannidae (15\%), Emberizidae $(6,11 \%)$ e Thraupidae $(5,55 \%)$. A família mais representativa entre as não-Passeriformes foi Columbidae (5\%). Em relação a FSM, as famílias mais representativas dos Passeriformes foram às mesmas que na FRP, com Tyrannidae $(18,85 \%)$, Emberizidae (6,55\%) e Thraupidae (5,74\%). Columbidae contou com 6,56\% de espécies observadas nessa área, sendo a família de não-Passeriformes mais representativa. A representatividade maior dos Tyrannidae registrada nas duas áreas concorda com outros estudos realizados na região tropical (DonATELl et al. 2004).

A riqueza específica das áreas analisadas está próxima aos valores obtidos em outros levantamentos realizados em matas do interior paulista. Pozza \& Pires (2003), trabalharam em dois

Revista Brasileira de Zoologia 24 (2): 362-375, junho 2007 
fragmentos de mata em Brotas (Estação Ecológica de São Carlos, 75ha) e Patrocínio Paulista (Fazenda Santa Cecília, 100ha), onde registraram respectivamente, 145 e 173 espécies. Donatelli et al. (2004) observaram 216 espécies na Fazenda Rio Claro, Lençóis Paulista (aproximadamente 1000ha).

Tanto a FRP como a FSM são cercadas por plantação de eucalipto que age diretamente sobre a composição e riqueza original das localidades amostrais. As matas sofrem ainda ação direta do efeito de borda, tais como aumento da luminosidade (podendo favorecer espécies de plantas invasoras), aumento da temperatura e invasão de espécies de aves que normalmente utilizam a borda da mata, competindo assim com espécies de interior de mata. Espécies de borda e interior podem responder a alterações na disponibilidade de habitats independentemente umas das outras, mas alterações entre esses grupos de espécies também podem implicar em fatores que contribuem no declínio de espécies do interior com a fragmentação (WIENs 1994).

A freqüência de ocorrência (F.O.) foi distribuída em quatro classes de intervalos (Tab. I).

Tabela I. Porcentagem das classes de freqüência de ocorrência da Fazenda Rio das Pedras (FRP) e Fazenda Santa Maria II (FSM).

\begin{tabular}{cccccc}
\hline & \multicolumn{5}{c}{ Classe de F.O. (\%) } \\
\cline { 3 - 6 } & & $1-25 \%$ & $26-50 \%$ & $51-75 \%$ & $76-100 \%$ \\
\hline \multirow{2}{*}{ FRP } & \% relativa & 42,3 & 24,5 & 11,6 & 21,6 \\
& $\mathrm{n}^{\circ}$ de espécies & 76 & 44 & 21 & 39 \\
\multirow{2}{*}{ FSM \% relativa } & 42,8 & 23,8 & 12,7 & 20,7 \\
& $\mathrm{n}^{\circ}$ de espécies & 54 & 30 & 16 & 26 \\
\hline
\end{tabular}

As espécies com 100\% de F.O., isto é, as que foram observadas em todas as visitas a FRP, correspondem à cerca de $8 \%$ das espécies amostradas, tais como Cyclarhis gujanensis (Gmelin, 1789); Thraupis sayaca (Linnaeus, 1766); Sporophila caerulescens (Vieillot, 1823); Pitangus sulphuratus (Linnaeus, 1766); Penelope superciliaris Temminck, 1815; Patagioenas picazuro (Temminck, 1813); e Coragyps atratus (Bechstein, 1793). Já na FSM, 12\% do total das espécies amostradas apresentaram F.O. = 100\%, como Coragyps atratus; Milvago chimachima (Vieillot, 1816); Patagioenas picazuro; Colaptes campestris (Vieillot, 1818); Thamnophilus caerulescens Vieillot, 1816; Platyrinchus mystaceus Vieillot, 1818; Pitangus sulphuratus; Chiroxiphia caudata (Shaw \& Nodder, 1793); Zonotrichia capensis (Statius Muller, 1776); Thraupis sayaca; Basileuterus culicivorus (Deppe, 1830); e Cyclarhis gujanensis.

De todas as espécies registradas, foram observadas em todas as visitas nas duas localidades amostrais: Coragyps atratus, Patagioenas picazuro, Thamnophilus caerulescens, Pitangus sulphuratus, Chiroxiphia caudada, Thraupis sayaca, Basileuterus culicivorus e Cyclarhis gujanensis. Essa baixa porcentagem de espécies com F.O = 100\% e a alta porcentagem com F.O menor que $50 \%$ pode representar um baixo índice de indivíduos nas popu- lações das espécies na comunidade, ou seja, as que utilizam o fragmento o ano todo e que tem capacidade de explorar o ambiente tanto para nidificação como na alimentação. Vários fatores podem ser a causa dessa baixa freqüência: espécies pouco conspícuas, ou seja, que possuem vocalização discreta ou que pouco vocalizam; de ocorrência ocasional, ou seja, que não são típicas do ambiente de estudo; de espécies migratórias, e/ou porque os remanescentes florestais não têm condições de manter populações por escassez de recursos alimentares. Segundo KARR (1977), espécies irregulares podem ser difíceis de interpretação, podendo ser raras por estarem em seu limite de tolerância fisiológica devido a algum fator ambiental ou físico, e/ou porque o recurso da qual dependem é raro na região. Um frugívoro especialista ou uma espécie que requer um local para nidificação especializado também pode ser raro se sua alimentação ou requerimento de habitat não for suficiente. Espécies irregulares também podem ser acidentais, vindas de outros habitats vizinhos.

As espécies comuns as duas áreas somam um total de $\mathrm{n}=$ 105 (Anexo I). Aplicando-se o índice de Sorensen obteve-se o valor de $C_{s}=0,67$, que representa uma considerável similaridade entre as áreas, apesar de os dois fragmentos apresentarem características fisionômicas diferentes como já citado anteriormente, o que permite a existência de microhabitats distintos, bem como a diferença na topografia entre as matas. As classes de freqüências não mostraram diferenças significativas entre as duas áreas ( $\mathrm{U}=12.00 \mathrm{~ns})$.

\section{Levantamento Quantitativo}

No levantamento quantitativo por pontos de escuta foram registradas 65 espécies na FRP e 64 espécies na FSM; isto corresponde, respectivamente, a $36 \%$ e $51 \%$ do valor encontrado no levantamento qualitativo. Aproximadamente $50 \%$ das espécies registradas neste levantamento foram observadas no segundo mês de amostragem nas duas fazendas. A partir de maio a curva cumulativa de espécies praticamente se estabilizou na FRP e em junho na FSM (Fig. 1).

O número de espécies registradas nas áreas amostrais não apresentou variação significativa ao longo do período amostral. Na FRP esteve entre 22 (março de 2005) e 37 (fevereiro de 2005), com coeficiente de variação de $17,32 \%$. Na FSM o coeficiente de variação foi de $18,24 \%$ sendo que em fevereiro de 2005 foi registrado o menor número de espécies $(n=18)$ e em novembro de 2004 o maior $(n=36)$.

Na FRP foram registrados 833 contatos, com média de cerca de 8,3 contatos/amostra. A média de contatos por mês variou de 5,8 (novembro de 2004) a 11,7 (fevereiro de 2005). Na FSM registraram-se 1027 contatos, com média de cerca de 10,2 contatos/amostra. A média de contatos por mês variou de 8,1 (setembro de 2004 e fevereiro e julho de 2005) a 13,9 (novembro de 2004).

As comunidades se mostraram diferentes nas duas áreas como descrito para o levantamento qualitativo. Essa diferença também na abundância indicada poderia ser atribuída às diferenças de entorno, à configuração espacial e à existência de 


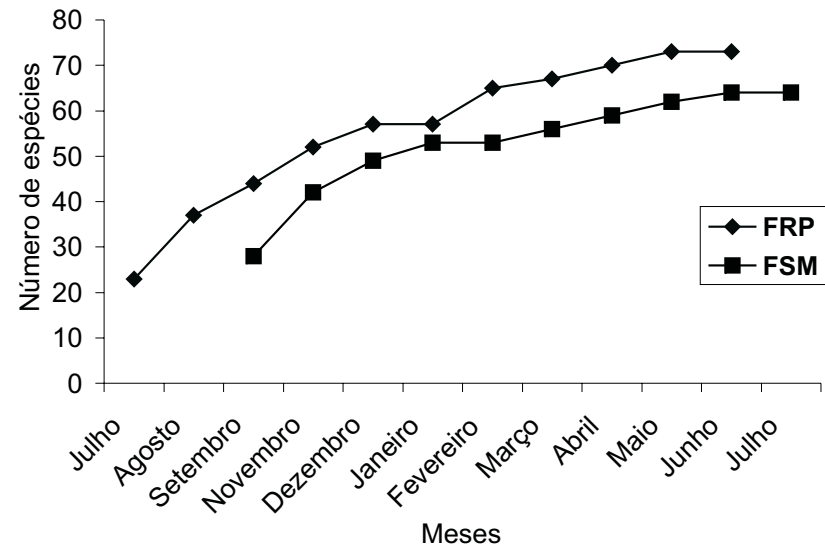

Figura 1. Curva cumulativa de espécies registradas pelo levantamento quantitativo na Fazenda Rio das Pedras (FRP) e na Fazenda Santa Maria II (FSM) ao longo do período amostral.

clareiras no interior da FRP. Isto pode ter excluído alguns microambientes necessários para certas espécies exclusivas de uma área ou outra.

A média de contatos por amostra pode ser considerada baixa quando comparada a outros estudos. Donatelli et al. (2004) registraram 12,7 contatos/amostra e LyrA-Neves et al. (2004) obtiveram uma média de $24,0 \pm 7,6$ contatos/amostra. Deve-se considerar que esses valores correspondem a fragmentos maiores que os da FRP e FSM. Quando as médias de contato/amostra das duas fazendas FRP e FSM são comparadas com trabalhos realizados em fragmentos menores como em Pozza \& PIREs (2003), que exploraram dois fragmentos (75 e 100ha) no interior paulista e registraram respectivamente 5,4 e 6,7 contatos/ amostra, observa-se uma proporcionalidade, tamanho do fragmento e contato/amostra nos resultados, quanto menor as frações de estudo, menores os contatos por amostra. Além disso, as duas localidades são isoladas de outros fragmentos florestais, podendo evitar a dispersão de espécies que necessitam de grandes áreas para alimentação.

Na FRP, o índice pontual de abundância apresentou variação de 0,01 (1 contato) a 1,140 (114 contatos), com uma média de 0,083 (equivalente a oito contatos/espécie). A curva cumulativa do número de espécies por ordem decrescente de abundância (Fig. 2) mostra que existem cerca de 41\% das espécies com índice de detecção maior ou igual à média ( $\mathrm{n}=8$ espécies), 59\% das espécies ( $\mathrm{n}=38$ ) com índice de detecção menor que a media de contatos. Já a FSM apresentou uma variação do índice pontual de abundância de 0,01 (1 contato) a 0,97 (97 contatos), com média de 0,16 (equivalente a 16 contatos/espécie). A curva cumulativa do número de espécies por ordem decrescente de abundância (Fig. 2) mostra que existem cerca de $33 \%$ das espécies com índice de detecção maior que a média (n = 21 espécies), sendo $67 \%$ das espécies $(n=43)$ com índice de detecção menor que a média de contatos. Em termos gerais pode-se dizer que poucas espécies são abundantes nas duas lo- calidades estudadas e que a maioria apresenta poucos indivíduos por espécie, perfil este registrado em ambientes de mata tropicais (STотz et al. 1996).

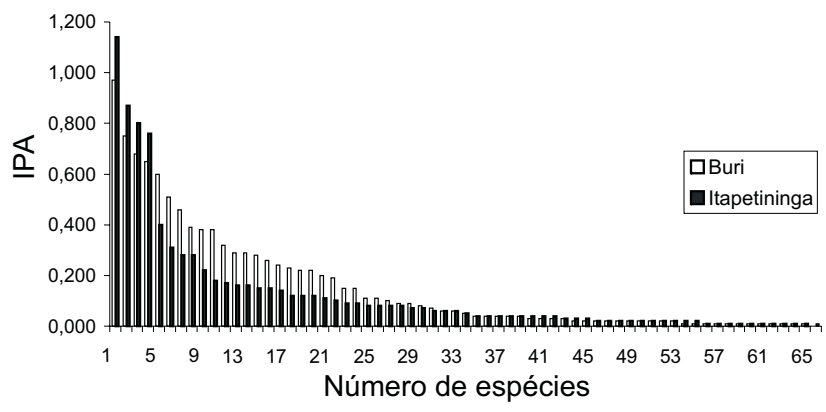

Figura 2. Valores decrescentes de IPA das espécies registradas nas Fazendas Rio das Pedras (FRP) e Santa Maria II (FSM) ao longo do período amostral.

As espécies mais abundantes registradas na FRP são: Patagioenas picazuro (IPA $=1,140)$, Chiroxiphia caudata $(\mathrm{IPA}=$ 0,870), Basileuterus culicivorus (IPA =0,800), e Cyclarhis gujanensis $(\mathrm{IPA}=0,760)$. Tais espécies representam cerca $43 \%$ do número total de contatos registrados. Na FSM, as espécies mais abundantes são: Patagioenas picazuro (IPA $=0,97$ ), Basileuterus leucoblepharus (Vieillot, 1817) (Parulidae) (IPA = 0,75), Cyclarhis gujanensis (IPA $=0,68)$, Corythopis delalandi (Lesson, 1830) (Tyrannidae) (IPA $=0,65)$ e Chiroxiphia caudata $(\mathrm{IPA}=0,60)$ e representam cerca $36 \%$ do número total de contatos registrados.

Pozza \& Pires (2003), no estudo de dois fragmentos, observaram que no primeiro fragmento o IPA variava de 0,008 (um contato) a 0,408 (47 contatos) e no segundo, de 0,008 (um contato) a 0,660 (76 contatos); Donatelli et al. (2004), registraram IPA variando de 0,001 (um contato) a 0,700 (53 contatos). Enquanto que Lyra-Neves et al. (2004) encontraram variação de 0,004 (um contato) a 1,263 (341 contatos). Estes trabalhos apresentam um perfil semelhante ao observado na FRP e na FSM, onde um número elevado de espécies apresenta baixos valores de IPA e um pequeno número apresenta abundância elevada, não importando o intervalo entre o maior e o menor valor. Esse é o padrão básico verificado em estudos utilizando o método de ponto de escuta.

Segundo KARR (1977) muitas espécies são raras devido a um conjunto de fatores que abrangem desde o tamanho das espécies correlacionado ao ambiente que está sendo estudado até associações realizadas com outros organismos na alimentação, como é o caso das espécies seguidoras de formigas.

Aplicando-se o índice de diversidade de Shannon-Wiener, obteve-se $\mathrm{H}^{\prime}=3,04$ para a FRP e $\mathrm{H}^{\prime}=2,85$ para a FSM. A variação deste índice foi de 2,62 (março de 2005) a 3,22 (fevereiro de 2005) na FRP e de 2,76 (fevereiro de 2005) a 3,20 (novembro de 2004) na FSM. Donatelli et al. (2004) encontraram H'= 
3,10 , em um fragmento de mata de 1000 ha no interior do estado de São Paulo. Constatou-se que, apesar de o fragmento da FRP ser pequeno, obteve um bom índice de diversidade comparado com os valores obtidos por Donatelli et. al. (2004). A eqüitatividade, assim como a diversidade, apresentou pouca variação ao longo do período amostral (valores próximos a 0,9) nas duas áreas, indicando que as espécies registradas estão distribuídas similarmente nas amostras.

\section{Categorias alimentares}

Pode-se observar que na FSM (Tab. II) pouco mais da metade de todas as espécies de aves registradas (53\%) são insetívoras. As espécies frugívoras (23\%) têm uma representação muito menor em relação às insetívoras, sendo que aproximadamente uma em cada quatro aves pertence a esta categoria alimentar. Evidenciou entretanto uma maior porcentagem em relação às onívoras (17\%). As espécies nectarívoras têm pouca representatividade (5\%) e são representadas basicamente por beija-flores. As espécies detritívoras foram representadas em sua totalidade por urubus, tendo a menor representatividade entre as categorias alimentares (2\%). Nenhuma espécie carnívora foi registrada no interior do remanescente florestal na FSM.

Na FRP, pode-se observar um quadro muito semelhante (Tab. II), tanto nas espécies quanto nos contatos registrados dentro de cada categoria alimentar. As espécies insetívoras representaram metade das espécies registradas (50\%), sendo as frugívoras (26\%) com uma representatividade pouco distinta em relação à da FSM; as aves frugívoras apresentaram também maior porcentagem que as onívoras (18\%). As espécies nectarívoras tiveram pouca representatividade $(5,5 \%)$, tendo sido representadas basicamente por beija-flores. As detritívoras tiveram $0,5 \%$, representadas exclusivamente por urubus. Como na FSM, nenhuma espécie de ave carnívora foi registrada no interior do remanescente florestal.

Em termos gerais pode-se dizer que a distribuição das categorias alimentares nos dois fragmentos de mata analisados segue um mesmo padrão nas duas localidades: maior porcentagem de aves insetívoras, seguidas de frugívoras, onívoras, nectarívoras e detritívoras. A cada duas aves registradas em cada uma das duas fazendas, uma era insetívora, sendo que a grande maioria destas eram compostas por aves Passeriformes. Foi encontrada uma porcentagem pouco maior de insetívoros Passeriformes na FSM $(87,9 \%)$ em relação a FRP (85\%), sendo Tyrannidae a família mais representativa nesta categoria na FSM e Thamnophilidae na FRP. Segundo Sick (1997), a alta porcentagem de aves insetívoras registradas é padrão para matas da região tropical. Outros autores também constataram a dominância dos Tyrannidae em relação às demais aves insetívoras, tais como Willis (1979), MotTa-Júnior (1990), Donatelli et al. (2004) e TELINO-JúNIOR et al. (2005). Em relação às aves frugívoras, constatou-se na FSM que a maioria das aves desta categoria são nãoPasseriformes (53,3\%), mas não em FRP (41\%). Possivelmente na FSM, ainda exista uma produção contínua de alimentos ao longo dos anos, para frugívoros de médio e grande porte, tais como Pionus maximiliani (Kuhl, 1820) e Penelope superciliaris Temminck, 1815, por exemplo. Na FRP registrou-se como frugívora de médio porte, Amazona aestiva (Linnaeus, 1758) (com IPA significativo) e de grande porte Penelope superciliaris como (IPA bem menor) (ver Anexo I). A presença de aves generalistas (onívoras) neste estudo não representa um grande percentual geral $(17,1 \%$ na FSM e $18 \%$ na FRP) e foi semelhante nas duas áreas tanto para os Passeriformes quanto para os não-Passeriformes. TELINO-JÚNIOR et al. (2005) registraram também um predomínio de aves insetívoras, mas seguidas de aves onívoras e não de frugívoras como relatamos neste trabalho. Tais resultados descritos pelos autores supracitados devem-se, sobretudo, pela análise de vários ambientes distintos na Reserva Estadual de Gurjaú, incluindo-se áreas abertas, enquanto que no presente estudo somente foi considerado o interior dos remanescentes florestais.

Não concordamos com Almeida (1982) quando afirma que o número de espécies onívoras em geral é maior que as insetívoras nas matas menos alteradas e o de insetívoras aumenta nas mais alteradas. Conforme salienta WiLLIS (1979), uma dieta mais variada como a de aves onívoras é favorecida em ambientes perturbados e não em ambientes menos perturbados. Assim AlmeidA (1982) não analisou os dados em termos de número de indivíduos em cada categoria trófica, mas o número de espécies de

Tabela II. Categorias alimentares registradas na comunidade de aves nas fazendas Santa Maria II (Buri) e Rio das Pedras (Itapetininga), Estado de São Paulo. (NP) aves não-Passeriformes; (P) Passeriformes; (T) Total.

\begin{tabular}{|c|c|c|c|c|c|c|c|c|c|c|c|c|}
\hline \multirow[t]{2}{*}{ Categorias } & \multicolumn{6}{|c|}{ Fazenda Santa Maria II } & \multicolumn{6}{|c|}{ Fazenda Rio das Pedras } \\
\hline & NP & $\%$ & $P$ & $\%$ & $\mathrm{~T}$ & $\%$ & NP & $\%$ & $P$ & $\%$ & $\mathrm{~T}$ & $\%$ \\
\hline Detritívoro & 1 & 100,0 & 0 & 0,0 & 1 & 2,0 & 1 & 100,0 & 0 & 0 & 1 & 0,5 \\
\hline Frugívoro & 8 & 53,3 & 7 & 46,7 & 15 & 23,0 & 7 & 41,0 & 10 & 59,0 & 17 & 26,0 \\
\hline Insetívoro & 4 & 12,1 & 30 & 87,9 & 34 & 53,0 & 5 & 15,0 & 28 & 85,0 & 33 & 50,0 \\
\hline Nectarívoro & 3 & 100 & - & 2,2 & 3 & 6,3 & 3 & 75,0 & 1 & 25,0 & 4 & 5,5 \\
\hline Carnívoro & - & - & - & - & - & - & - & - & - & - & - & - \\
\hline Onívoro & 3 & 27,3 & 8 & 72,7 & 11 & 17,1 & 3 & 25,0 & 9 & 75,0 & 12 & 18,0 \\
\hline Totais & 19 & 29,7 & 45 & 70,3 & 64 & 100,0 & 19 & 28,3 & 48 & 71,7 & 67 & 100,0 \\
\hline
\end{tabular}


uma forma geral, o que modificou a interpretação dos resultados desse autor. Em fragmentos com tamanho reduzido, as aves onívoras podem estar em maior porcentagem que as frugívoras (Willis 1979), o que certamente não é o caso deste estudo em que foi registrado um maior número de aves frugívoras de pequeno, médio e grande porte em ambos fragmentos.

O baixo percentual de onívoros, representados basicamente na copa e borda, sugere que a borda da mata não avançou significativamente nos fragmentos de matas das fazendas estudadas, como ocorre em matas onde há acentuado processo de desmatamento periférico (DonAtelli et al. 2004). Em relação às demais categorias, observou-se um baixo valor para aves detritívoras, pois estas foram registradas pousadas nas copas ou apenas passando pelas matas, sendo o valor obtido perfeitamente compatível.

A ausência de aves carnívoras nas duas matas é preocupante já que era esperado o registro de aves de rapina no interior de tais fragmentos, particularmente de gaviões e corujas, como registrado por outros autores que estudaram comunidades de aves em fragmentos de mata (Willis 1979). Este autor afirma que a perda de gaviões e outras aves de rapina no interior de remanescentes florestais deve-se à diminuição populacional e a extinções pontuais naqueles fragmentos de tamanho reduzido (em seu estudo, seria < que 250 ha). Tais aves são aquelas que estão entre as mais susceptíveis à fragmentação e, portanto, seriam mais vulneráveis à extinção local, pois são de grande porte, especializadas, têm baixa tolerância ao habitat matriz, baixa densidade e baixa taxa de sobrevivência anual (SIEVING \& KARR 1997).

Donatelli et al. (2004), estudando a comunidade de aves da Fazenda Rio Claro, Lençóis Paulista, São Paulo, constatou que há mais aves de espécies insetívoras no interior da mata que externa a ela, sendo esta comunidade insetívora do interior da mata composta na sua maioria por aves Passeriformes, um indício de preservação. SEKercioglu et al. (2002) afirma que a fragmentação florestal atinge mais as aves insetívoras que as granívoras ou onívoras, não obstante a abundância de animais invertebrados das quais tais aves insetívoras se alimentam. O autor constatou que a riqueza e a abundância das espécies insetívoras são significativamente menores quanto menor for o fragmento.O declínio populacional das aves insetívoras está associado a predação de ninhos e a efeitos estocásticos. O estudo de Donatelli et al. (2004) aponta que a presença de uma quantidade significativa de aves frugívoras de grande porte no interior da mata (aproximadamente uma em cada três frugívoros eram de grande porte) corrobora a hipótese de mata preservada, uma vez que o ambiente possui condições de manter espécies que se alimentam de frutos durante o ano todo.

Muitas aves adaptadas a ambientes abertos podem adentrar o interior da mata em fragmentos pequenos e até ocupar todo o seu interior (Goosem 1997). Segundo Lovejoy et al. (1986), as espécies de aves que invadem fragmentos normalmente possuem alta habilidade de dispersão e são generalistas quanto à alimentação e à utilização de habitat, ao contrário daquelas que vivem no interior florestal. No caso do presente estudo, observou-se uma menor porcentagem de aves onívoras que as insetívoras e frugívoras, fato este que indicaria uma relativa estabilidade dos fragmentos analisados.

Em relação ao parâmetro categoria alimentar, de uma forma geral, as análises de riqueza e abundância evidenciaram um padrão para as áreas amostradas, o que poderia indicar ou sugerir que tais fragmentos apresentam uma relativa estabilidade: 1) aves insetívoras dominantes nos dois remanescentes; 2) presença de aves frugívoras, de médio e grande porte, em segundo lugar em ambos fragmentos; 3) menor porcentagem de aves generalistas; e 4) constatou-se também que não houve diferença significativa entre as categorias alimentares nos dois fragmentos estudados (insetívoros $(\mathrm{U}=6.000, \mathrm{~ns})$; frugívoros ( $U=10.00$, ns) e onívoros $(U=21.00, n s)$ ).

\section{Estratificação}

Na FSM (Tab. III), observou-se que as aves insetívoras são mais representativas no sub-bosque (53\%), as frugívoras apresentaram pouco mais da metade dos contatos no sub-bosque (50\%), as nectarívoras dividiram-se em copa (50\%) e sub-bosque (50\%), o que já era esperado uma vez que a maioria dos representantes desta categoria é constituída por beija-flores. As aves onívoras apresentaram uma distribuição equilibrada entre copa $(45,5 \%)$ e sub-bosque $(45,5 \%)$. Todos os detritívoros registrados forrageiam no solo (100\%), pois foram representados por urubus.

Na FRP (Tab. III), a representatividade de aves insetívoras é ligeiramente maior também no sub-bosque (51,4\%). As aves frugívoras foram mais evidentes na copa $(52,6 \%)$. Nas aves nectarívoras, a distribuição foi equilibrada, metade sub-bosque (50\%) e metade copa (50\%). Em relação às aves onívoras, a distribuição do estrato ocupado foi equilibrada, sendo registrada um pouco mais da metade dos contatos desta categoria na copa $(54,5 \%)$ e pouco menos no sub-bosque $(45,5 \%)$. Assim como na FSM, toda as aves detritívoras registradas forrageiam no solo.

As aves insetívoras são mais representativas no sub-bosque nas duas áreas de estudo, fato este que corrobora a boa preservação das duas áreas estudadas, uma vez que boa parte destas aves é exclusiva de mata (Sick 1997). Como foi observado por BierregaARd \& STOUfFer (1997), as aves de sub-bosque são aquelas que permanecem nos fragmentos e por esta razão são mais vulneráveis às alterações antrópicas.

Os insetívoros especialistas de estrato vertical correspondem a $14,7 \%$ na FSM e a $16,2 \%$ na FRP e compreendem as famílias Picidae e Dendrocolaptidae. Segundo Sick (1997), a preservação das matas é essencial para a existência das aves insetívoras de estrato vertical, já que o reflorestamento com eucaliptos e Pinus não favorece a existência dos pica-paus (Picidae), o mesmo acontecendo com as capoeiras nativas, nas quais faltam árvores maiores para a instalação de seus ninhos. Os Dendrocolaptidae estão entre os Passeriformes mais abundantes em matas neotropicais, sobretudo em florestas primárias; entretanto, em matas secundárias empobrecidas, com reduzido número de espécies vegetais e de árvores maiores e velhas, 
Tabela III. Categorias alimentares registradas e estratos ocupados pelas aves nas fazendas Santa Maria II (Buri) e Rio das Pedras (Itapetininga), Estado de São Paulo.

\begin{tabular}{|c|c|c|c|c|c|c|c|c|}
\hline & \multicolumn{4}{|c|}{ Fazenda Santa Maria II } & \multicolumn{4}{|c|}{ Fazenda Rio das Pedras } \\
\hline & Copa & Solo & Sub-bosque & Vertical & Copa & Solo & Sub-bosque & Vertical \\
\hline Detritívoros & - & 100,0 & - & - & 100,0 & & - & - \\
\hline Frugívoros & 35,7 & 14,3 & 50,0 & - & 52,6 & 21,1 & 26,3 & - \\
\hline Insetívoros & 26,5 & 5,8 & 53,0 & 14,7 & 32,4 & - & 51,4 & 16,2 \\
\hline Nectarívoros & 50,0 & - & 50,0 & - & 50,0 & - & 50,0 & - \\
\hline Onívoros & 45,5 & 9,0 & 45,5 & - & 54,5 & - & 45,5 & 1,0 \\
\hline
\end{tabular}

seu número reduz-se significativamente (SICK 1997).

As aves frugívoras de copa e de solo também são vulneráveis as alterações antrópicas, incluindo-se neste caso uma disponibilidade de alimentos distribuídos ao longo do ano. As aves frugívoras de copa participam de bandos mistos de forrageamento, observados muitas vezes durante a coleta de dados. Já as espécies de solo são geralmente de porte médio, como aquelas pertencentes ao gênero Crypturellus e à família Columbidae. Foram observados também nestas áreas estudadas alguns frugívoros de grande porte, pertencentes às famílias Cracidae e Psittacidae, o que sugere que as referidas áreas estudadas possuem condições de manter espécies que se alimentam de frutos durante todo o ano, incluindo as de grande porte.

A porcentagem de onívoros de copa, tais como Trogon surrucura (Vieillot, 1817), indica uma exclusividade no hábito alimentar dessas aves no referido estrato, pois são aves que não são encontradas fora da mata. Segundo WiLlis (1979), alterações ambientais podem levar a uma tendência ao aumento de aves onívoras e possivelmente de insetívoras menos especializadas e decréscimo de frugívoras e insetívoras mais especializadas.

Os nectarívoros de sub-bosque podem sofrer influência do tamanho da área, tais como os Trochilidae que necessitam de áreas maiores para dispersão (BierRegaARd \& Stouffer 1997). No caso das duas áreas amostradas, observou-se que os nectarívoros não estão bem representados, o que pode indicar que o pequeno tamanho das matas é um fator preocupante para boa conservação destes ambientes.

Embora os fragmentos encontrem-se isolados e circundados por vegetação de eucalipto, observa-se uma estabilidade da avifauna local, tanto pelo valor da eqüidistribuição quanto pelo índice de diversidade e pela riqueza. Contudo, é necessário que haja ações de conservação e monitoramento destas áreas, não só por estarem isoladas, mas também pelas freqüentes ações antrópicas sofridas e pequenas áreas que ocupam tais fragmentos.

\section{AGRADECIMENTOS}

À Duratex S/A, Itapetininga e Buri, em nome de José L. da S. Maia, diretor de meio ambiente, pela oportunidade de desenvolvimento do projeto nas dependências da empresa, e aos funcionários pela ajuda e atenção durante o desenvolvimento do mesmo.

Revista Brasileira de Zoologia 24 (2): 362-375, junho 2007

\section{REFERÊNCIAS BIBLIOGRÁFICAS}

Almeida, A.F. 1982. Análise das categorias de nichos tróficos das aves de matas ciliares em Anhembi, Estado de São Paulo. Silvicultura em São Paulo 16 (3): 1787-1795.

BierregaArd Jr, R.O. \& P.C. Stouffer.1997. Understory birds and dynamic habitat mosaics in amazonian rainforest, p.138155.In: W.F.Laurance \& R.O. BierregaARd JR (Eds). Tropical forest remnants: ecology, management and conservation of fragmented communities. Chicago, The University of Chicago Press, XV+616p.

Bierregatrd Jr, R.O. \& T.E. Lovejoy. 1989. Effects of florest fragmentation an American understory bird communities. Acta Amazonica 19: 215-241,

Bierregaard Jr, R.O.; T.E. Lovejoy; V. Kapos; A.A. dos Santos \& R.W. Hutchings. 1992. The biological dynamics of tropical rainforest fragments. BioScience 42: 859-866.

Collar, N.J.; L.P.Gonzaga; N. Krabbe; A. Madronõ Nieto; L.G. NARANJo; T.A PARKER III \& D.C.Wege. 1992. Threatened birds of Americas. Washington, The ICBP/IUCN Red Data Book, $3^{\text {rd }}$ ed., 1150p.

DANIELSEN, F. 1997. Stable environments and fragile communities: does history determine the resilence of avian rain-forest communities to habitat degradation? Biodiversity and Conservation 6: 423-433.

Donatelli, R.J.; T.V.V. Costa \& C.D. Ferreira. 2004. Dinâmica da avifauna em fragmento de mata na Fazenda Rio Claro, Lençóis Paulista, São Paulo, Brasil. Revista Brasileira de Zoologia 21 (1): 97-114.

Goosem, M. 1997. Internal fragmentation: the effects of roads highways, and powerline clearings on movements and mortality of rainforest vertebrates, p. 241-255. In: W.F. LAURENCE \& R.O. BIERREGAARD (Eds). Tropical forest remmants: ecology, management and conservation of fragment comminities. Chicago, The University of Chicago Press, 632p.

JARVIS, P.J. 1994. Environmental changes, p. 42-85. In: R.W. FurNESS \& J.J.D. GREenWoOD (Eds). Birds as monitor of environmental change. London, Cahpman and Hall, 368p.

KARR, J.R. 1977. The impact of nearstream vegetation and stream morphology on walter quality andstream biota. Springfield, U.S. Office of Research and Development, 83p. KREBS, C.J. 1999. Ecological methodology. Los Angeles, Benjamins 
Cammings, $2^{\text {nd }}$ ed., 620p.

Lovejoy, T. E.; R.O. Bierregard; A.B. Rylands Jr; J.R. Malcom; C.E. Quintela; L.H. Harper; K.S. Brown Jr; A.H. Powell; G.N.V. Powell; H.O.R. Schubart \& M. HaYs. 1986. Edge and other effects of isolation of Amazon forests fragmentations, p. 257285. In: M.E. SOULÉ (Ed). Conservation biology: the science of scarcity and diversity. Sunderland, Sinauer, 584p.

Lyra-Neves, R.M.; de M.M. Dias; S.M. de Azevedo-Júnior; W.R. Telino-Júnior \& M.E.L. De LarrazÁbal. 2004. Comunidade de aves da Reserva Estadual de Gurjaú, Pernambuco, Brasil. Revista Brasileira de Zoologia 21 (3): 581-592.

MotTA-JúnIOR, J.C. 1990. Estrutura trófica e composição das avifaunas de três habitats terrestres na região central do estado de São Paulo. Ararajuba 1: 65-71.

Pozza, D.D. \& J.S.R. Pires. 2003. Avifauna em dois fragmentos de floresta estacional semidecídua do interior paulista. Brazilian Journal of Biology 63 (2): 307-319.

Serercioglu, C.H.; P.R.Ehrlich; G.C. Daily; D. Aygen; D. Goehring $\&$ R.F. SANDI. 2002. Disappearance of insectivorous birds from tropical forest fragments. Proceedings National Academy Sciences 99: 263-267.

SICK, H. 1997. Ornitologia brasileira. Rio de Janeiro, Novo Fronteira, 912p.

Sieving, K.E. \& J.R. KarR. 1997. Avian extinction and persistence mechanisms in lowland Panama, p.138-155. In: W.F. LAURANCE \& R.O. BieRREgAaRd JR. (Eds). Tropical forest remnants: ecology, management and conservation of fragmented communities. Chicago, The University of Chicago Press, 632p.

Stotz, D.F.; J.W. Fitzpatrick; T.A. Parker \& D.K. Moskovits. 1996, Neotropical birds. Ecology and conservation. Chicago, University of Chicago Press, 502p.

Telino-Júnior, W.R.; M.M. Dias; S.M. Júnior; R.M. Lyra-Neves \& M.E.L. LARRAŹ́bal. 2005. Estrutura trófica da avifauna na Reserva Estadual de Gurjaú, Zona da Mata Sul, Pernambuco, Brasil. Revista Brasileira de Zoologia 22 (4): 962-973.

Temple, S. A. \& J.A.Wiens, 1989. Bird population and environmental change: Can be bio-indicators? American Birds, Washington, 43:260-270.

Verner, J. 1981. Measuring responses of avian communities to habitat manipulation. Studies in Avian Biology 6: 543-547.

Wiens, J.A. 1994. Habitat fragmentation: island v landscape perspectives on bird conservation. The Ibis 137: 97-104,

Wilcox, B.A. \& D.D. Murphy. 1985. Conservation strategy: the effects of fragmentation on extinction. The American Naturalist 125: 879-887.

Willis, E.O. 1979. The composition of avian communities in remanescent woodlots in southern Brazil. Papéis Avulsos de Zoologia 33 (1): 1-25.

Zorzetto, R.; C. Fioravanti \& M. Ferroni, 2003. A floresta renasce. Revista FAPESP 91: 10-23.

Recebido em 02.VIII.2006; aceito em 02.V.2007.

Anexo I. Frequência de ocorrência (F.O.) e índice pontual de abundância (IPA) das espécies de aves das Fazendas Rio das Pedras (FRP), Itapetininga, e Santa Maria (FSM), Buri.

\begin{tabular}{|c|c|c|c|c|}
\hline \multirow{2}{*}{ Espécies } & \multicolumn{2}{|c|}{ FRP } & \multicolumn{2}{|c|}{ FSM } \\
\hline & F.O. (\%) & IPA & F.O. (\%) & IPA \\
\hline \multicolumn{5}{|l|}{ Tinamidae } \\
\hline Crypturellus obsoletus (Temminck, 1815) & & & 40 & 0,030 \\
\hline Crypturellus parvirostris (Wagler, 1827) & 10 & & & \\
\hline Rhynchotus rufescens (Temminck, 1815) & 20 & & & \\
\hline \multicolumn{5}{|l|}{ Anatidae } \\
\hline Dendrocygna viduata (Linaeus, 1766) & 20 & & & \\
\hline Sarkidiornis sylvicola lhering \& ihering, 1907 & 10 & & & \\
\hline Amazonetta brasiliensis (Gmelin, 1789) & 70 & & & \\
\hline \multicolumn{5}{|l|}{ Cracidae } \\
\hline Penelope superciliaris Temminck, 1815 & 100 & 0,060 & 40 & 0,010 \\
\hline \multicolumn{5}{|l|}{ Phasianidae } \\
\hline Odontophorus capueira (Spix, 1825) & & & 20 & 0,020 \\
\hline \multicolumn{5}{|l|}{ Phalacrocoracidae } \\
\hline Phalacrocorax brasillianus (Gmelin, 1789) & 30 & & & \\
\hline \multicolumn{5}{|l|}{ Anhingidae } \\
\hline Anhinga anhinga Linnaeus, 1766 & 30 & & & \\
\hline
\end{tabular}


Anexo I. Frequência de ocorrência (F.O.) e índice pontual de abundância (IPA) das espécies de aves das Fazendas Rio das Pedras (FRP), Itapetininga, e Santa Maria (FSM), Buri.

\begin{tabular}{|c|c|c|c|c|}
\hline \multirow{2}{*}{ Espécies } & \multicolumn{2}{|c|}{ FRP } & \multicolumn{2}{|c|}{ FSM } \\
\hline & F.O. (\%) & IPA & F.O. (\%) & IPA \\
\hline \multicolumn{5}{|l|}{ Ardeidae } \\
\hline Tigrisoma lineatum (Boddaert, 1753) & 20 & & & \\
\hline Butorides striatus (Linnaeus, 1758) & 20 & & & \\
\hline Ardea cocoi Linnaeus, 1766 & 50 & & & \\
\hline Ardea alba Linnaeus, 1758 & 80 & & & \\
\hline Syrigma sibilatrix (Temminck, 1824) & 60 & & 40 & \\
\hline \multicolumn{5}{|l|}{ Threskiornithidae } \\
\hline Platalea ajaja Linnaeus, 1758 & 20 & & & \\
\hline \multicolumn{5}{|l|}{ Cathartidae } \\
\hline Cathartes aura (Linnaeus, 1758) & 50 & & 50 & \\
\hline Coragyps atratus (Bechstein, 1793) & 100 & 0,040 & 100 & 0,010 \\
\hline Sarcoramphus papa (Linnaeus, 1758) & 10 & & & \\
\hline \multicolumn{5}{|l|}{ Acciptridae } \\
\hline Leptodon cayanensis (Latham, 1790) & 20 & & & \\
\hline Elanus leucurus (Vieillot, 1818) & 30 & & & \\
\hline Rosthramus sociabilis (Vieillot, 1817) & 10 & & & \\
\hline Ictinia plumbea (Gmelin, 1758) & 20 & & & \\
\hline Heterospizias meridionalis (Latham, 1790) & 10 & & 10 & \\
\hline Rupornis magnirostris (Gmelin, 1788) & 80 & & 80 & \\
\hline \multicolumn{5}{|l|}{ Falconidae } \\
\hline Caracara plancus (Miller, 1777) & 100 & & 70 & \\
\hline Milvago chimachima (Vieillot, 1816) & 70 & & 100 & \\
\hline Herpetotheres cachinnans (Linnaeus, 1758) & 40 & & & \\
\hline Micrastur ruficollis (Vieillot, 1817) & & & 10 & \\
\hline Falco sparverius Linnaeus, 1758 & 10 & & 30 & \\
\hline Falco femoralis Temminck, 1822 & 10 & & & \\
\hline \multicolumn{5}{|l|}{ Rallidae } \\
\hline Aramides saracura (Spix, 1825) & & & 10 & \\
\hline Gallinula chloropus (Linnaeus, 1758) & 40 & & & \\
\hline \multicolumn{5}{|l|}{ Cariamidae } \\
\hline Cariama cristata (Linnaeus, 1766) & 40 & & 20 & \\
\hline \multicolumn{5}{|l|}{ Charadriidae } \\
\hline Vanellus chilensis (Molina, 1782) & 90 & & 60 & \\
\hline \multicolumn{5}{|l|}{ Recurvirostridae } \\
\hline Himantopus melanurus Vieillot, 1817 & 30 & & & \\
\hline \multicolumn{5}{|l|}{ Scolopacidae } \\
\hline Tringa flavipes (Gmelin, 1789) & 10 & & & \\
\hline Tringa solitaria Wilson, 1813 & 10 & & & \\
\hline \multicolumn{5}{|l|}{ Jacanidae } \\
\hline Jacana jacana (Linnaeus, 1766) & 60 & & & \\
\hline \multicolumn{5}{|l|}{ Columbidae } \\
\hline Columbina minuta (Linnaeus, 1766) & 10 & & & \\
\hline Columbina talpacoti (Temminck, 1811) & 90 & 0,010 & 90 & \\
\hline
\end{tabular}


Anexo I. Continuação.

\begin{tabular}{|c|c|c|c|c|}
\hline \multirow{2}{*}{ Espécies } & \multicolumn{2}{|c|}{ FRP } & \multicolumn{2}{|c|}{ FSM } \\
\hline & F.O. (\%) & IPA & F.O. (\%) & IPA \\
\hline Columbina squammata (Lesson, 1831) & 100 & & 20 & \\
\hline Columbina picui (Temminck, 1813) & & & 10 & \\
\hline Columba livia Gmelin, 1789 & & & 10 & \\
\hline Patagioenas picazuro (Temminck, 1813) & 100 & 1,140 & 100 & 0,970 \\
\hline Patagioenas cayennensis (Bonnaterre, 1792) & 20 & 0,040 & & \\
\hline Zenaida auriculata (Des Murs, 1847) & 80 & 0,020 & 80 & \\
\hline Leptotila verreauxi Bonaparte, 1855 & 60 & 0,100 & 50 & 0,060 \\
\hline Leptotila rufaxilla (Richard \& Bernard, 1792) & 30 & 0,040 & 20 & 0,020 \\
\hline Geotrygon montana (Linnaeus, 1758) & 30 & 0,020 & & \\
\hline \multicolumn{5}{|l|}{ Psittacidae } \\
\hline Aratinga leucophthalma (Statius Muller, 1776) & 30 & & 10 & 0,020 \\
\hline Forpus xanthopterygius (Spix, 1824) & 10 & & & \\
\hline Brotogeris tirica (Gmelin, 1788) & 10 & & & \\
\hline Pionus menstrus (Linnaeus, 1766) & & & 10 & \\
\hline Pionus maximiliani Kuhl, (1820) & 20 & & 10 & 0,020 \\
\hline Amazona aestiva (Linnaeus, 1758) & 20 & 0,020 & & \\
\hline \multicolumn{5}{|l|}{ Cuculidae } \\
\hline Piaya cayana (Linnaeus, 1766) & 70 & 0,020 & 50 & 0,040 \\
\hline Crotophaga ani Linnaeus, 1758 & 60 & & 70 & \\
\hline Guira guira (Gmelin, 1788) & 60 & & 40 & \\
\hline Tapera naevia (Linnaeus, 1766) & 10 & & & \\
\hline \multicolumn{5}{|l|}{ Tytonidae } \\
\hline Tyto alba (Scopoli, 1769) & 20 & & 10 & \\
\hline \multicolumn{5}{|l|}{ Strigidae } \\
\hline Megascops choliba (Vieillot, 1817) & 10 & & 30 & \\
\hline Bubo virginianus (Gmelin, 1788) & 10 & & & \\
\hline Athene cunicularia (Molina, 1782) & 10 & & 70 & \\
\hline \multicolumn{5}{|l|}{ Caprimulgidae } \\
\hline Lurocalis semitorquatus (Gmelin, 1789) & & & 30 & 0,030 \\
\hline Nyctidromus albicollis (Gmelin, 1789) & 20 & & 20 & \\
\hline Nyctiphrynus ocellatus (Tschudi, 1844) & 10 & & & \\
\hline Caprimulgus rufus Boddaert, 1783 & 10 & & & \\
\hline Caprimulgus parvulus Gould, 1837 & 10 & & & \\
\hline Hydropsalis torquata (Gmelin, 1789) & 20 & & & \\
\hline \multicolumn{5}{|l|}{ Apodidae } \\
\hline Streptoprocne zonaris (Shaw, 1796) & & & 20 & \\
\hline \multicolumn{5}{|l|}{ Trochilidae } \\
\hline Phaethornis pretrei (Lesson \& Delattre, 1839) & 30 & 0,010 & & 0,020 \\
\hline Phaethornis eurynome (Lesson, 1832) & 10 & & & \\
\hline Eupetonema macroura (Gmelin, 1788) & 20 & & 10 & \\
\hline Florisuga fusca (Vieillot, 1817) & 10 & & & \\
\hline Chlorostilbon lucidus (Shaw, 1812) & 10 & & & \\
\hline Thalurania glaucopis (Gmelin, 1788) & 20 & 0,010 & 20 & 0,020 \\
\hline
\end{tabular}


Anexo I. Continuação.

\begin{tabular}{|c|c|c|c|c|}
\hline \multirow{2}{*}{ Espécies } & \multicolumn{2}{|c|}{ FRP } & \multicolumn{2}{|c|}{ FSM } \\
\hline & F.O. (\%) & IPA & F.O. (\%) & IPA \\
\hline Hylocharis chrysura (Shaw, 1812) & 60 & & 10 & 0,010 \\
\hline Leucochloris albicollis (Vieillot, 1818) & 10 & & 20 & \\
\hline \multicolumn{5}{|l|}{ Trogonidae } \\
\hline Trogon surrucura Vieillot, 1817 & 80 & 0,150 & 60 & 0,150 \\
\hline \multicolumn{5}{|l|}{ Alcedinidae } \\
\hline Ceryle torquatus (Linnaeus, 1766) & 40 & & & \\
\hline Choroceryle amazona (Latham, 1790) & 30 & & & \\
\hline Choroceryle americana (Gmelin, 1788) & 10 & & & \\
\hline \multicolumn{5}{|l|}{ Ramphastidae } \\
\hline Ramphastos toco Statius Muller, 1776 & 50 & 0,050 & & \\
\hline \multicolumn{5}{|l|}{ Picidae } \\
\hline Picumnus temminckii Lafresnaye, 1845 & 10 & & & \\
\hline Picumnus albosquamatus d'Orbigny, 1840 & 90 & 0,120 & 80 & 0,200 \\
\hline Melanerpes candidus (Otto, 1796) & 10 & 0,020 & 20 & \\
\hline Veniliornis spilogaster (Wagler, 1827) & 40 & 0,080 & & \\
\hline Colaptes melanochloros (Gmelin, 1788) & 20 & & & \\
\hline Colaptes campestris (Vieillot, 1818) & 80 & 0,010 & 100 & \\
\hline Celeus flavescens (Gmelin, 1788) & 20 & 0,020 & 50 & 0,040 \\
\hline Dryocopus lineatus (Linnaeus, 1766) & 50 & & 30 & 0,020 \\
\hline \multicolumn{5}{|l|}{ Thamnophilidae } \\
\hline Mackenziaena severa (Lichtenstein, 1823) & 30 & & 10 & 0,010 \\
\hline Thamnophilus doliatus (Linnaeus, 1764) & & & 10 & 0,010 \\
\hline Thamnophilus punctatus (Shaw, 1809) & 30 & & & \\
\hline Thamnophilus caerulescens Vieillot, 1816 & 100 & 0,400 & 100 & 0,460 \\
\hline Dysithamnus mentalis (Temminck, 1823) & 60 & & 100 & 0,320 \\
\hline Herpsilochmus rufumarginatus (Temminck, 1822) & 20 & 0,010 & & \\
\hline Drymphila ochropyga (Hellmayr, 1906) & 10 & & & \\
\hline Drymophila malura Temminck, 1825 & 30 & 0,020 & & \\
\hline Pyriglena leucoptera (Vieillot, 1818) & 100 & 0,280 & 20 & \\
\hline \multicolumn{5}{|l|}{ Conopophagidae } \\
\hline Conopophaga lineata (Wied, 1831) & 90 & 0,140 & 90 & 0,230 \\
\hline \multicolumn{5}{|l|}{ Rhynocryptidae } \\
\hline Scytalopus speluncae (Ménétriès, 1835) & & & 10 & \\
\hline Scytalopus indigoticus (Wied, 1831) & & & 40 & 0,050 \\
\hline \multicolumn{5}{|l|}{ Dendrocolaptidae } \\
\hline Sittasomus griseicapillus (Vieillot, 1818) & 100 & 0,310 & 80 & 0,190 \\
\hline Xiphorhynchus fuscus (Vieillot, 1818) & & & & 0,290 \\
\hline Lepidocolaptes angustirostris (Vieillot, 1818) & 10 & & 80 & \\
\hline \multicolumn{5}{|l|}{ Furnariidae } \\
\hline Furnarius rufus (Gmelin, 1788) & 90 & & 50 & \\
\hline Synallaxis ruficapilla Vieillot, 1819 & 90 & 0,280 & 80 & 0,280 \\
\hline Synallaxis spixi Sclater, 1856 & 60 & & 50 & 0,010 \\
\hline Certhiaxis cinnamomeus (Gmelin, 1788) & 20 & & & \\
\hline Automolus leucophthalmus (Wied, 1821) & 50 & 0,040 & 100 & 0,380 \\
\hline
\end{tabular}


Anexo I. Continuação.

\begin{tabular}{|c|c|c|c|c|}
\hline \multirow{2}{*}{ Espécies } & \multicolumn{2}{|c|}{ FRP } & \multicolumn{2}{|c|}{ FSM } \\
\hline & F.O. (\%) & IPA & F.O. (\%) & IPA \\
\hline Lochmias nematura (Lichtenstein, 1823) & & & 50 & 0,110 \\
\hline Xenops rutilans Temminck, 1821 & 20 & & 10 & \\
\hline \multicolumn{5}{|l|}{ Tyrannidae } \\
\hline Corythopis delalandi (Lesson, 1830) & 60 & 0,150 & 90 & 0,650 \\
\hline Poecilotriccus plumbeiceps (Lafresnaye, 1846) & 10 & & 10 & 0,010 \\
\hline Poecilotriccus latirostris (Pelzeln, 1868) & 10 & 0,010 & 10 & 0,010 \\
\hline Todirostrum cinereum (Linnaeus, 1766) & & & 20 & 0,020 \\
\hline Elaenia flavogaster (Thunberg, 1822) & 80 & & 70 & \\
\hline Camptostoma obsoletum (Temminck, 1824) & 50 & 0,020 & 50 & 0,070 \\
\hline Serpophaga subcristata (Vieillot, 1817) & 10 & & 10 & 0,010 \\
\hline Tolmomyias sulphurescens (Spix, 1825) & 10 & 0,010 & 30 & 0,010 \\
\hline Platyrinchus mystaceus Vieillot, 1818 & 80 & 0,120 & 100 & 0,290 \\
\hline Myiophobus fasciatus (Statius Muller, 1776) & 20 & & & \\
\hline Lathrotriccus euleri (Cabanis, 1868) & 60 & 0,110 & 70 & 0,220 \\
\hline Contopus cinereus (Spix, 1825) & & & 20 & \\
\hline Pyrocephalus rubinus (Bossaert, 1753) & 10 & & & \\
\hline Satrapa icterophrys (Vieillot, 1818) & 10 & & & \\
\hline Xolmis cinereus (Vieillot, 1816) & 20 & & 60 & \\
\hline Xolmis velata (Lichtenstein, 1823) & 40 & & 40 & \\
\hline Gubernetes yetapa (Vieillot, 1818) & 20 & & & \\
\hline Colonia colonus (Vieillot, 1818) & 50 & 0,010 & & \\
\hline Machetornis rixosus (Vieillot, 1819) & 50 & & 10 & \\
\hline Legatus leucophaius (Vieillot, 1818) & 20 & & 20 & \\
\hline Myiozetetes similis (Spix, 1825) & 40 & & 10 & \\
\hline Pitangus sulphuratus (Linnaeus, 1766) & 100 & 0,060 & 100 & 0,030 \\
\hline \multicolumn{5}{|l|}{ Philohydor lictor (Lichtenstein, 1823) } \\
\hline Myiodynastes maculatus (Statius Muller, 1776) & 10 & & 50 & 0,110 \\
\hline Megarhynchus pitangua (Linnaeus, 1766) & 70 & 0,040 & 50 & 0,900 \\
\hline Tyrannus melancholicus Vieillot, 1819 & 60 & 0,040 & 60 & 0,040 \\
\hline Tyrannus savanna Vieillot, 1808 & 60 & & 50 & \\
\hline Myiarchus swainsoni Cabanis \& Heine, 1859 & 30 & 0,010 & 10 & \\
\hline Myiarchus ferox (Gmelin, 1789) & 60 & 0,020 & & \\
\hline Pachyramphus polychopterus (Vieillot, 1818) & & & 70 & 0,240 \\
\hline Pachyramphus validus (Lichtenstein, 1823) & 30 & & 10 & 0,010 \\
\hline \multicolumn{5}{|l|}{ Cotingidae } \\
\hline Procnias nudicollis (Vieillot, 1817) & & & 40 & \\
\hline Pyroderus scutatus (Shaw, 1792) & 10 & & 20 & \\
\hline \multicolumn{5}{|l|}{ Pipridae } \\
\hline Chiroxiphia caudata (Shaw \& Nodder, 1793) & 100 & 0,870 & 100 & 0,600 \\
\hline Schiffornis virescens (Lafresnaye, 1838) & 10 & & 20 & \\
\hline \multicolumn{5}{|l|}{ Vireonidae } \\
\hline Cyclarhis gujanensis (Gmelin, 1789) & 100 & 0,760 & 100 & 0,680 \\
\hline Vireo olivaceus (Linnaeus, 1766) & 60 & 0,170 & 50 & 0,220 \\
\hline Hylophilus poicilotis Temminck, 1822 & 20 & 0,010 & & \\
\hline
\end{tabular}


Anexo I. Continuação.

\section{Espécies}

\section{Corvidae}

Cyanocorax cristatellus (Temminck, 1823)

Cyanocorax chrysops (Vieillot, 1818)

Hirundinidae

Tachycineta albiventer (Boddaert, 1783)

Progne chalybea (Gmelin, 1789)

Pygochelidon cyanoleuca (Vieillot, 1817)

Stelgidopteryx ruficollis (Vieillot, 1817)

Troglodytidae

Troglodytes musculus Naumann, 1823

Turdidae

Platycichla flavipes (Vieillot, 1818)

Turdus rufiventris Vieillot, 1818

Turdus leucomelas Vieillot, 1818

Turdus amaurochalinus Cabanis, 1851

Turdus albicollis Vieillot, 1818

\section{Mimidae}

Mimus saturninus (Lichtenstein, 1823)

\section{Coerebidae}

Coereba flaveola (Cabanis, 1851)

Thraupidae

Thlypopsis sordida (d'Orbigny \& Lafresnaye, 1837)

Cypsnagra hirundinacea (Lesson, 1831)

Trichothraupis melanops (Vieillot, 1818)

Piranga flava (Vieillot, 1822)

Habia rubica (Vieillot, 1817)

Tachyphonus coronatus (Vieillot, 1822)

Thraupis sayaca (Linnaeus, 1766)

Pipraedea melanonota (Vieillot, 1819)

Tangara cayana (Linnaeus, 1766)

Tersina viridis (Illiger, 1811)

Dacnis cayana (Linnaeus, 1766)

Emberizidae

Zonotrichia capensis (Statius Muller, 1776)

Ammodramus humeralis (Bosc, 1792)

Emberizoides herbicola (Vieillot, 1817)

Volatinia jacarina (Linnaeus, 1766)

Sporophila lineola (Linnaeus, 1758)

Sporophila caerulescens (Vieillot, 1823)

Sporophila bouvreuil (Statius Muller, 1776)

Coryphospingus cucullatus (Statius Muller, 1776)

Saltator fuliginosus (Daudin, 1800)

Saltator similis d'Orbigny \& Lafresnaye, 1837

Saltator atricollis Vieillot, 1817

\begin{tabular}{cccccc} 
& & & \multicolumn{2}{c}{ FSM } \\
\cline { 1 - 1 } \cline { 5 - 5 } F.O. (\%) & IPA & & F.O. (\%) & IPA \\
80 & 0,030 & & 20 & \\
40 & 0,040 & & 50 & 0,030
\end{tabular}

10

60

90

90

40

40

80

60

0,160

0,180

0,020

30

0,030

0,380

0,060

0,010

10

0,040

100

80

0,120

0,150

10

0,010

20

20

30

0,070

0,090

0,070

0,260

0,100

100

10

90

$0,160 \quad 10$

$0,030 \quad 10$

70

50

100

10

60

10

60

100

10

40

10

40

30
0,020

0,080 
Anexo I. Continuação.

\begin{tabular}{|c|c|c|c|c|}
\hline \multirow{2}{*}{ Espécies } & \multicolumn{2}{|c|}{ FRP } & \multicolumn{2}{|c|}{ FSM } \\
\hline & F.O. (\%) & IPA & F.O. (\%) & IPA \\
\hline \multicolumn{5}{|l|}{ Parulidae } \\
\hline Parula pitiayumi (Vieillot, 1817) & 30 & & 10 & \\
\hline Geothlypis aequinoctialis (Gmelin, 1789) & 20 & & & \\
\hline Basileuterus culicivorus (Deppe, 1830) & 100 & 0,800 & 100 & 0,510 \\
\hline Basileuterus flaveolus (Baird, 1865) & 40 & 0,080 & & \\
\hline Basileuterus leucoblepharus (Vieillot, 1817) & 10 & & 100 & 0,750 \\
\hline \multicolumn{5}{|l|}{ Icteridae } \\
\hline Psarocolius decumanus (Pallas, 1769) & 80 & 0,220 & 10 & \\
\hline Chrysomus ruficapillus Vieillot, 1819 & 10 & & & \\
\hline Pseudoleistes guirahuro (Vieillot, 1819 & 50 & & 20 & \\
\hline Molothrus bonariensis (Gmelin, 1798) & 20 & & & \\
\hline \multicolumn{5}{|l|}{ Fringilidae } \\
\hline Carduelis magelanica (Vieillot, 1805) & 80 & & 10 & \\
\hline Euphonia chlorotica (Linnaeus, 1766) & 80 & 0,090 & 50 & 0,040 \\
\hline
\end{tabular}

